\title{
1 Carbon-13 NMR Relaxation Study of the Internal Dynamics in Cyclodextrins in Isotropic 2 Solution
}

\author{
Piotr Bernatowicz, ${ }^{*},{ }^{\dagger}$ Katarzyna Ruszczyńska-Bartnik, ${ }^{\ddagger}$ Andrzej Ejchart,${ }^{\ddagger}$ Helena Dodziuk, ${ }^{\dagger}$ \\ Ewa Kaczorowska, ${ }^{\ddagger}$ and Haruhisa Ueda ${ }^{\S}$ \\ Institute of Physical Chemistry, Polish Academy of Sciences, Kasprzaka 44/52, 01-224 Warsaw, Poland, \\ Institute of Biochemistry and Biophysics, Pawińskiego 5a, 02-106 Warsaw, Poland, and Department of Physical \\ Chemistry, Hoshi University, 4-41 Ebara 2-chome, Shinagawa-ku, Tokyo 142-8501, Japan
}

Received: September 2, 2009; Revised Manuscript Received: November 5, 2009

\begin{abstract}
${ }^{13} \mathrm{C}$ nuclear spin relaxation processes in seven cyclodextrins (from six-membered $\alpha$ to twelve-membered $\eta$ ) were investigated in ${ }^{2} \mathrm{H}_{2} \mathrm{O}$ solution at multiple magnetic fields. Detailed analysis of ${ }^{13} \mathrm{C}$ longitudinal relaxation in laboratory and rotating frames and ${ }^{13} \mathrm{C}\left\{{ }^{1} \mathrm{H}\right\}$ nuclear Overhauser enhancement in these molecules yielded their rotational diffusion tensors and a semiquantitative picture of their internal dynamics.
\end{abstract}

\section{Introduction}

For more than 30 years macrocyclic oligosugars-cyclodextrins, $\mathrm{CDs}^{1}$ - were considered to have a rigid, truncated-cone structure. ${ }^{2,3}$ Such a structure of $C_{n}$ symmetry $(n=6$ for $\alpha-C D, 1,7$ for $\beta$-CD, 2 , etc.) with all glycosidic oxygen atoms lying in a plane and forming a regular polygon stemmed from X-ray studies, although the analyses of native $\mathrm{CD}$ geometries $(n=6-8)$ using this technique did not show such a high symmetry, and only averaged parameters for their structure are given in the reviews by Harata. ${ }^{4,5}$ This opinion on the rigidity of native CDs contradicts model considerations, since the macrocycles are built of relatively rigid glucopyranose units connected by the glycosidic bonds characterized by a low barrier of $1 \mathrm{kcal} / \mathrm{mol}$ to internal rotation. ${ }^{6}$ Model molecular mechanics calculations on $\mathbf{1}$ yielded asymmetric structures corresponding to broad energy minima. $^{7}$

Considering CDs as rigid molecules did not change even after discovery of the flip-flop mechanism interchanging the direction of the $\mathrm{O} 2 \mathrm{H}$ and $\mathrm{O} 3 \mathrm{H}$ hydrogen bonds in the macrocycles. ${ }^{4} \mathrm{On}$ the other hand, ${ }^{1} \mathrm{H}$ and ${ }^{13} \mathrm{C}$ NMR spectra of $\mathrm{CDs}$ in liquids display chemical shift equivalence of corresponding protons and carbons of glucopyranose units, pointing either to highly symmetrical structures or to the intramolecular motions fast on the chemical shift time scale. ${ }^{8}$ The first possibility is incompatible with the disappearance of the broad $v_{\mathrm{OH}}$ band at ca. 3400 $\mathrm{cm}^{-1}$ in the Raman spectra of microcrystalline $\beta-\mathrm{CD} \cdot 12 \mathrm{H}_{2} \mathrm{O}$ after exposure to ${ }^{2} \mathrm{H}_{2} \mathrm{O}$ or $\mathrm{H}_{2}{ }^{17} \mathrm{O}$ in view of the obstacles posed by the densely packed crystal lattice to the rapid movement of water molecules. This movement could be feasible only by structural fluctuations of the CD molecules temporarily opening appropriate diffusion paths. ${ }^{9}$ The crystallization of $\mathbf{1}$ (and also $\mathbf{2}$ and 3) with different amounts of water molecules ${ }^{4,5}$ as well as the facility of complexation of guest molecules of various shapes ${ }^{1}$ would not be possible without a considerable degree of CD flexibility. The nonrigidity of CD complexes and the guest mobility inside the CD cavity was in discussed in detail in refs 2 and 3. In particular, two cases of substituted CDs were

* To whom the correspondence should be addressed. E-mail: bernat@ ichf.edu.pl.

${ }^{\dagger}$ Polish Academy of Sciences.

* Institute of Biochemistry and Biophysics.

$\S$ Hoshi University. reported, for which the internal motions of the macrocycles were at least partly frozen at low temperature manifested by a considerable broadening and/or splitting of NMR signals. ${ }^{10,11}$

In spite of the finally recognized CD flexibility, no experimental attempt was undertaken to provide a specific picture of their internal mobility in solution. The NMR relaxation studies performed by Kowalewski et al., ${ }^{12,13}$ although originally aiming at investigations of the $\mathrm{CD}$ dynamics, interpreted the experimental results in terms of the Lipari-Szabo formalism. ${ }^{14}$ This approach is easy in implementation since it does not require any physical picture of the internal dynamics, yet as a result, it delivers, apart from the global correlation time, the values of generalized order parameters, $S^{2}$, the physical meaning of which is not precisely defined, and values of effective correlation times characterizing intramolecular motions. The latter quantities absorb the discrepancies between the relaxation parameters measured and calculated on the basis of an assumed model of global molecular reorientation, so that, by definition, they do not provide insight into a real molecular dynamics. This is a serious limitation of the Lipari-Szabo analysis. The present study attempts to fill this gap. It demonstrates that nuclear spin relaxation methods supported by pertinent theoretical models do allow for efficient simultaneous investigations of both global and internal molecular dynamics.

In the present investigations, only the ${ }^{13} \mathrm{C}$ relaxation rates of the ring carbon atoms, measured under conditions of proton decoupling, are exploited. The use of such a limited experimental data set is deliberate. Under the above conditions, the longitudinal relaxation behavior of the ${ }^{13} \mathrm{C}$ nucleus in a $\mathrm{CH}$ grouping is monoexponential ${ }^{15}$ so that error-prone decomposition of the recovery curves into single-exponential components is avoided. Moreover, the observed relaxation rate is almost completely dominated by the dipolar $\mathrm{C}-\mathrm{H}$ interaction within the grouping and is independent of cross-correlations with other timedependent interactions present in the system. In this way, difficult questions about the strenghts of the latter need not be addressed. It must also be stressed that the ${ }^{1} \mathrm{H}$ relaxation data are virtually useless for the present study. Apart from the already mentioned problems with evaluation of the relevant interaction strengths, the possible exploitation of the proton data is hampered also by the fact that in CDs the proton resonances come in partially overlapping multiplets. The individual com- 
92 ponents of the latter can in general relax with different rates 93 for which closed-form expressions are nonexistent. By and large, 94 dipolar proton-proton interactions within the same glucopy95 ranose ring do not exceed much the corresponding interactions 96

between the neighboring rings. Thus, the network of interactions that needs to be taken into account for an adequate description of the relaxation behavior of the ring protons is too extensive even for treatment in terms of the Redfield relaxation matrix.

\section{Experimental Section}

$\alpha-, \beta$-, and $\gamma-\mathrm{CD}$ (Sigma) were used without further purification. The large-ring $\mathrm{CDs}, \delta-\eta$, were obtained as described in ref 16. NMR samples of $650 \mu \mathrm{L}$ contained $10 \mathrm{mM} \mathrm{CD}$ solutions in ${ }^{2} \mathrm{H}_{2} \mathrm{O}$ (Armar Chemicals). ${ }^{1} \mathrm{H}$ and ${ }^{13} \mathrm{C}$ resonance assignments of methine groups were done de novo from 2D DQF-COSY and ${ }^{1} \mathrm{H} /{ }^{13} \mathrm{C}$ HSQC spectra (Supporting information). All chemical shifts in heteronuclear NMR spectra were reported with respect to external DSS- $d_{4}$. Chemical shifts of ${ }^{13} \mathrm{C}$ signals were assigned indirectly using the ratio of the zero-point frequencies, $f\left({ }^{13} \mathrm{C}\right) / f\left({ }^{1} \mathrm{H}\right)=0.251449530 .{ }^{17}$

The ${ }^{13} \mathrm{C}$ longitudinal relaxation rates in the laboratory $\left(R_{1 \mathrm{C}}\right)$ and rotating $\left(R_{1 \rho \mathrm{C}}\right)$ frames and ${ }^{13} \mathrm{C}\left\{{ }^{1} \mathrm{H}\right\}$ nuclear Overhauser enhancements $\left(\eta_{\mathrm{C}}\right)$ were measured at magnetic fields of 11.7, 9.4, and 7.0 T, using Varian Unity Plus 500 MHz, Varian Unity Inova $400 \mathrm{MHz}$, and Bruker Avance II $300 \mathrm{MHz}$ spectrometers, respectively. The temperature was set at $300.6 \mathrm{~K}$ in all experiments.

Both relaxation rates were determined using a series of $2 \mathrm{D}$ double INEPT based experiments with sensitivity enhancement adapted to ${ }^{13} \mathrm{C}$ from ${ }^{15} \mathrm{~N}$ sequences. ${ }^{18}$ The original $R_{2}$ experiment was adopted to $R_{1 \rho}$ measurements by substitution of the CPMG sequence with the spin-lock train of contiguous $180^{\circ}$ pulses on ${ }^{13} \mathrm{C}$ with alternating phases. ${ }^{19}$ The amplitudes of the spin-lock field were $1.66,1.33$, and $3.84 \mathrm{kHz}$ at $11.7,9.4$, and $7.0 \mathrm{~T}$, respectively. The $R_{1 \mathrm{C}}$ and $R_{1 \rho \mathrm{C}}$ data were obtained using ten evolution delays within the range of $0-55 \mathrm{~ms}$ and seven evolution delays within the range of 5-26 ms, respectively. Appropriate ${ }^{1} \mathrm{H} 180^{\circ}$ pulses were used during evolution of relaxation to suppress the effect of dipolar/CSA cross-correlation. ${ }^{20}$ The recycle delays were always longer than 5 times the longest proton $T_{1}$, usually exceeding $2 \mathrm{~s}$. The steady-state heteronuclear ${ }^{13} \mathrm{C}\left\{{ }^{1} \mathrm{H}\right\}$ NOEs were determined as a ratio of cross-peak intensities in two experiments, with and without proton presaturation. The appropriate sequence was taken from ref 19 and optimized for ${ }^{13} \mathrm{C}$. Since NOE measurements started from ${ }^{13} \mathrm{C}$ magnetization, the recycle delay had to be longer than 10 times the carbon $T_{1}$, typically $5.1 \mathrm{s.}^{21}$

Each 2D spectrum was acquired with $512\left(t_{2}\right) \times 128\left(t_{1}\right)$ complex data points with four transients per increment. Spectral widths were $1900 \mathrm{~Hz}$ in the ${ }^{1} \mathrm{H}$ dimension and 8000 $\mathrm{Hz}$ in the ${ }^{13} \mathrm{C}$ dimension. Zero filling was performed prior to the Fourier transformation. Data were processed using the program nmrPipe ${ }^{22}$ and analyzed with the program SPARKY. ${ }^{23}$ Resonance intensities were used in calculating relaxation times and NOE values. All the experiments were repeated at least twice. Experimental errors of the relaxation rates were obtained from appropriate elements of the variance-covariance matrix. Experimental errors in NOE values were evaluated from the formula $\sigma_{\mathrm{NOE}}=\left(1+\eta_{\mathrm{C}}\right)\left[(S / N)_{\mathrm{s}}{ }^{-2}+(S / N)_{\mathrm{ns}}{ }^{-2}\right]^{1 / 2}$, where $(S / N)_{\mathrm{s}}$ and $(S / N)_{\mathrm{ns}}$ denote signal-to-noise ratios in ${ }^{1} \mathrm{H}$ saturated and ${ }^{1} \mathrm{H}$ nonsaturated spectra, respectively. ${ }^{24}$ Experimental errors of NOE obtained in such a way were very close to those derived from the analysis of 10 separate measurements performed for $\mathbf{6}$ at $7.0 \mathrm{~T}$.
All the spectrometers were equipped with variable-temperature units allowing for temperature control with an accuracy of $0.1 \mathrm{~K}$. Temperature calibration was carefully performed using an ethylene glycol chemical shift thermometer. ${ }^{25}$ Both the gas flow and decoupling power were carefully controlled ${ }^{26}$ to diminish temperature gradients in the samples. Spectrometers were stabilized for at least $2 \mathrm{~h}$ before the beginning of the measurements.

Components of cyclodextrin rotational diffusion tensors (RDTs) were obtained from a least-squares iterative analysis of the relaxation data performed using a Fortran routine written in-house, based on the Newton-Raphson algorithm. ${ }^{27}$

\section{Results and Discussion}

References 28 and 29 contain a formalism necessary to link the molecular dynamics, both global and local, to spectral densities, $J(\omega)$, the impact of which on NMR observables, such as the ${ }^{13} \mathrm{C}$ longitudinal relaxation rate, $R_{1 \mathrm{C}},{ }^{13} \mathrm{C}-{ }^{1} \mathrm{H}$ crossrelaxation rate, $\rho_{\mathrm{C}}$, and relaxation rate in a rotating frame, $R_{1 \rho \mathrm{C}}$, is well established: ${ }^{15,30-33}$

$$
\begin{aligned}
R_{1 \mathrm{C}} & =T_{1 \mathrm{C}}{ }^{-1}=\frac{1}{20}\left[J\left(\omega_{\mathrm{H}}-\omega_{\mathrm{C}}\right)+3 J\left(\omega_{\mathrm{C}}\right)+6 J\left(\omega_{\mathrm{H}}+\omega_{\mathrm{C}}\right)\right] \\
\rho_{\mathrm{C}} & =\frac{\eta_{\mathrm{C}} \gamma_{\mathrm{C}} R_{1 \mathrm{C}}}{\gamma_{\mathrm{H}}}=\frac{1}{20}\left[6 J\left(\omega_{\mathrm{H}}+\omega_{\mathrm{C}}\right)-J\left(\omega_{\mathrm{H}}-\omega_{\mathrm{C}}\right)\right]
\end{aligned}
$$

$$
\begin{aligned}
R_{1 \rho \mathrm{C}}= & T_{1 \rho \mathrm{C}}^{-1}=\frac{1}{40}\left[\left(4 \sin ^{2} \xi\right) J\left(\omega_{1}\right)+\right. \\
(2- & \left.\sin ^{2} \xi\right) J\left(\omega_{H}-\omega_{C}\right)+\left(6-3 \sin ^{2} \xi\right) J\left(\omega_{\mathrm{C}}\right)+ \\
& \left.\left(6 \sin ^{2} \xi\right) J\left(\omega_{\mathrm{H}}\right)+6\left(2-\sin ^{2} \xi\right) J\left(\omega_{\mathrm{H}}+\omega_{\mathrm{C}}\right)\right]
\end{aligned}
$$

155 156 157
In eqs $1-3 \omega_{\mathrm{H}}$ and $\omega_{\mathrm{C}}$ denote ${ }^{1} \mathrm{H}$ and ${ }^{13} \mathrm{C}$ Larmor frequencies, respectively. $\xi$ is defined by $\tan \xi=\omega_{1} / \Delta$, where $\omega_{1}$ is the amplitude of the radio frequency field expressed in angular frequency units and $\Delta$ is the offset frequency for a given ${ }^{13} \mathrm{C}$ nucleus.

Since proton-decoupled ${ }^{13} \mathrm{C}$ spectra of all $\mathrm{CDs}$ under investigation contain in liquids only six signals manifesting high symmetry of the average NMR Hamiltonian, we assume the global motions of these molecules to be represented by axially symmetric RDTs with $D_{x}$ and $D_{y}$ equal and denoted by $D_{\perp}$ while the distinct $D_{z}$ component is further denoted by $D_{\|}$. As a mechanism for the local dynamics we assume diffusion of a glucopyranose unit about the axis interconnecting its glycosidic oxygen atoms, $\mathrm{O} 1$ and $\mathrm{O} 4$ (see Figure 1).

Since refs 28 and 29 do not comprise such a mechanism of the internal motion, we describe it by discrete dynamics taking place in very tiny steps. Populations of individual conformers assumed in this process are weighted by Gaussian-shaped distributions. Unfortunately, the exchange rates of interconversion of these conformers, which influence the most general eq 12 of ref 28 and eq 14.2 of ref 29 , are not known. We avoid this problem by assuming that the local dynamics is either very slow or very fast in comparison to the global molecular reorientation. In such cases the spectral densities of interest are given by

$$
J_{\text {slow }}(\omega)=\sum_{i=1}^{N} p_{i} D_{i, \mathrm{CH}}{ }^{2} \sum_{r=0}^{2} A_{r i}{ }^{2} \frac{2 \tau_{r}}{1+\left(\omega \tau_{r}\right)^{2}}
$$


Internal Dynamics in Cyclodextrins

$$
J_{\text {fast }}(\omega)=\tilde{D}_{\mathrm{CH}}{ }^{2} \sum_{r=0}^{2} \tilde{A}_{r}^{2} \frac{2 \tau_{r}}{1+\left(\omega \tau_{r}\right)^{2}}
$$

206

207 where

208

209 and

a)
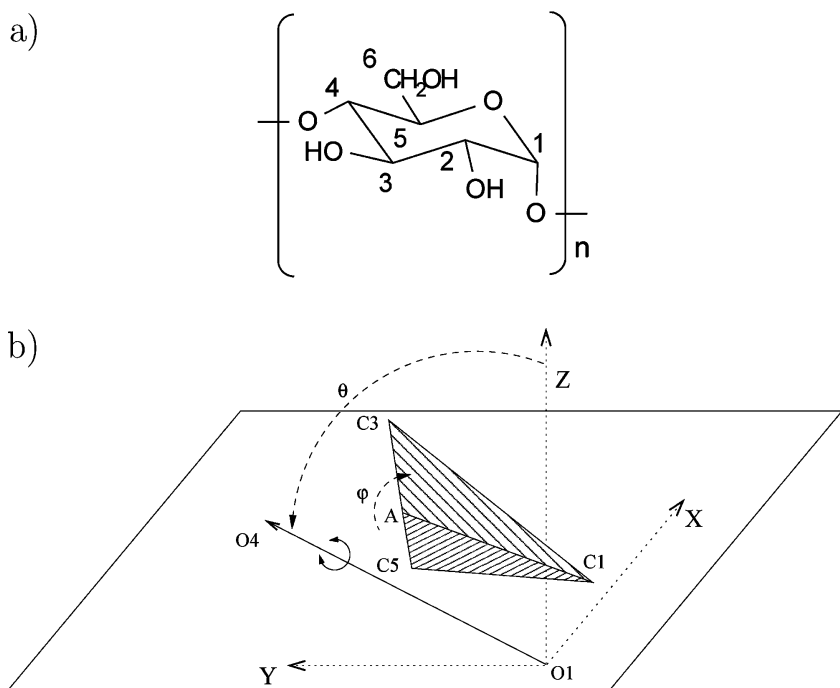

Figure 1. (a) $\alpha-, \beta-, \ldots, \eta$-CDs, $1-7$ ( $n=6-12$, respectively), and the atom numbering in the glucopyranose unit. (b) Schematic drawing of the orientation of a single glucopyranose unit in the reference frame of the RDT of a $\mathrm{CD}$ molecule. The segment $\mathrm{A}-\mathrm{C} 1$ is the intersection of the triangle spanned by $\mathrm{C} 1, \mathrm{C} 3$, and $\mathrm{C} 5$ atoms with the $x y$ plane of the RDT, coplanar with the average plane of glycosidic oxygen atoms. The angle between the $\mathrm{C} 1-\mathrm{C} 3-\mathrm{C} 5$ plane and the $x y$ plane of the RDT is denoted by $\varphi$. The angle between the $z$ axis of the RDT and the $\mathrm{O} 1-\mathrm{O} 4$ axis is denoted by $\theta .=$ The geometry of the $\mathrm{C} 1-\mathrm{C} 3-\mathrm{C} 5$ plane with respect to the $\mathrm{O} 1-\mathrm{O} 4$ axis is fixed; all these atoms are constituents of the glucopyranose unit assumed to be rigid. The double arrow depicts the internal dynamics, i.e., diffusion of the glucopyranose unit about the $\mathrm{O} 1-\mathrm{O} 4$ axis. Populations of the conformers arising as the result of this process are described by a Gaussian function whose width at half-height is given by the GHW parameter listed in column 1 of Table 1 .
J. Phys. Chem. B, Vol. xxx, No. xx, XXXX C

conclusions concerning a single unit are representative for all the units in a CD molecule.

In most instances relaxation of ${ }^{13} \mathrm{C}$ nuclei is realized by two main mechanisms: modulation of the dipolar interaction to the neighboring proton nuclei and modulation of their own chemical shielding anisotropies (CSAs). Since the CSA of aliphatic $\mathrm{CH}$ carbons of CDs is small, ca. $30-40$ ppm, ${ }^{34}$ we can safely neglect the latter mechanism at magnetic fields of 7.0-11.7 T. The error caused by this approximation is estimated to be not larger than about $2 \%$. Possible interference of dipolar and CSA mechanisms has been suppressed with ${ }^{1} \mathrm{H}$ pulses (see the Experimental Section).

To calculate the $D_{\mathrm{CH}}$ values, which are needed for the description of dipolar relaxation, neutron diffraction geometries of CDs are exploited, both native ${ }^{35-38}$ and in complexes. ${ }^{39-41}$ The procedure is as follows. For each $\mathrm{CH}$ bond in a single glucopyranose unit its mean length was calculated. The averaging was carried out over all the units whose neutron geometries had been found in refs $35-41$. Then we used the neutron geometry of an arbitrary glucopyranose unit, including both its glycosidic oxygens, but the lengths of all $\mathrm{CH}$ bonds were adjusted to calculated averages by shifting the proton positions along the respective $\mathrm{CH}$ bonds. The long-range $D_{\mathrm{CH}}$ values were then calculated directly from such a geometry, while one-bond $D_{\mathrm{CH}}$ values were further corrected; i.e., they were multiplied by so-called vibrational correction, ${ }^{42} \xi$. The magnitude of this correction, which was assumed to be the same for all one-bond $D_{\mathrm{CH}}$ values, was fitted simultaneously with other parameters in a numerical routine. The remaining parameters adjusted to "best fit" the measured relaxation data were the components of the RDTs, $D_{\|}$and $D_{\perp}$, and two angles, $\alpha$ and $\beta$, positioning the glucopyranose unit in the RDT coordinate frame. These angles do not have absolute sense. They describe the equilibrium orientation in the RDT coordinate frame of the glucopyranose unit, determined in the iterative minimization procedure, relative to an initial, arbitrary orientation of the CD molecule concerned, specified in the program input. Precisely, the equilibrum orientation is obtained as an effect of two rotations of the glucopyranose unit, first by angle $\alpha$ about axis $z$ and then by angle $\beta$ around axis $y$ of the RDT frame.

The analyzed data include both $R_{1 \mathrm{C}}$ values and $\rho_{\mathrm{C}}$ values. The former often have 1 order of magnitude larger values than the latter, so that the influence of both these data types on the results might not be comparable. Therefore, prior to the analysis, the individual $R_{1 \mathrm{C}}$ values and $\rho_{\mathrm{C}}$ values are weighted by the reciprocal of the averages of the respective quantities calculated for each $\mathrm{CD}$, ensuring a similar impact on the results.

The $R_{1 \rho \mathrm{C}}$ data, although they had been acquired, were not exploited at this stage for the following reason: the $\xi$ entering eq 3 is a function of the frequency offset, $\Delta$, which may vary for each ${ }^{13} \mathrm{C}$ nucleus among conformers along with their chemical shifts. Since the latter quantities for individual conformers are not known, we are not able to calculate the $R_{1 \rho \mathrm{C}}$ in the slow local dynamics regime. The situation is different in the fast local dynamics regime, where eq 3 requires $\xi$ averaged over all conformers, so that the knowledge of the individual chemical shifts for consecutive conformers is not required. Since the discrimination between fast and slow local dynamics had to be done, the $R_{1 \rho \mathrm{C}}$ values were rejected to compare identically processed data sets. Ultimately, they were calculated a posteriori (see below). For the slow local dynamics regime averaged chemical shifts were used perforce, so that the resulting values should be considered with caution. 
TABLE 1: Results of Numerical Iterative Analysis Which Passed the Selection Procedure Described in the Text

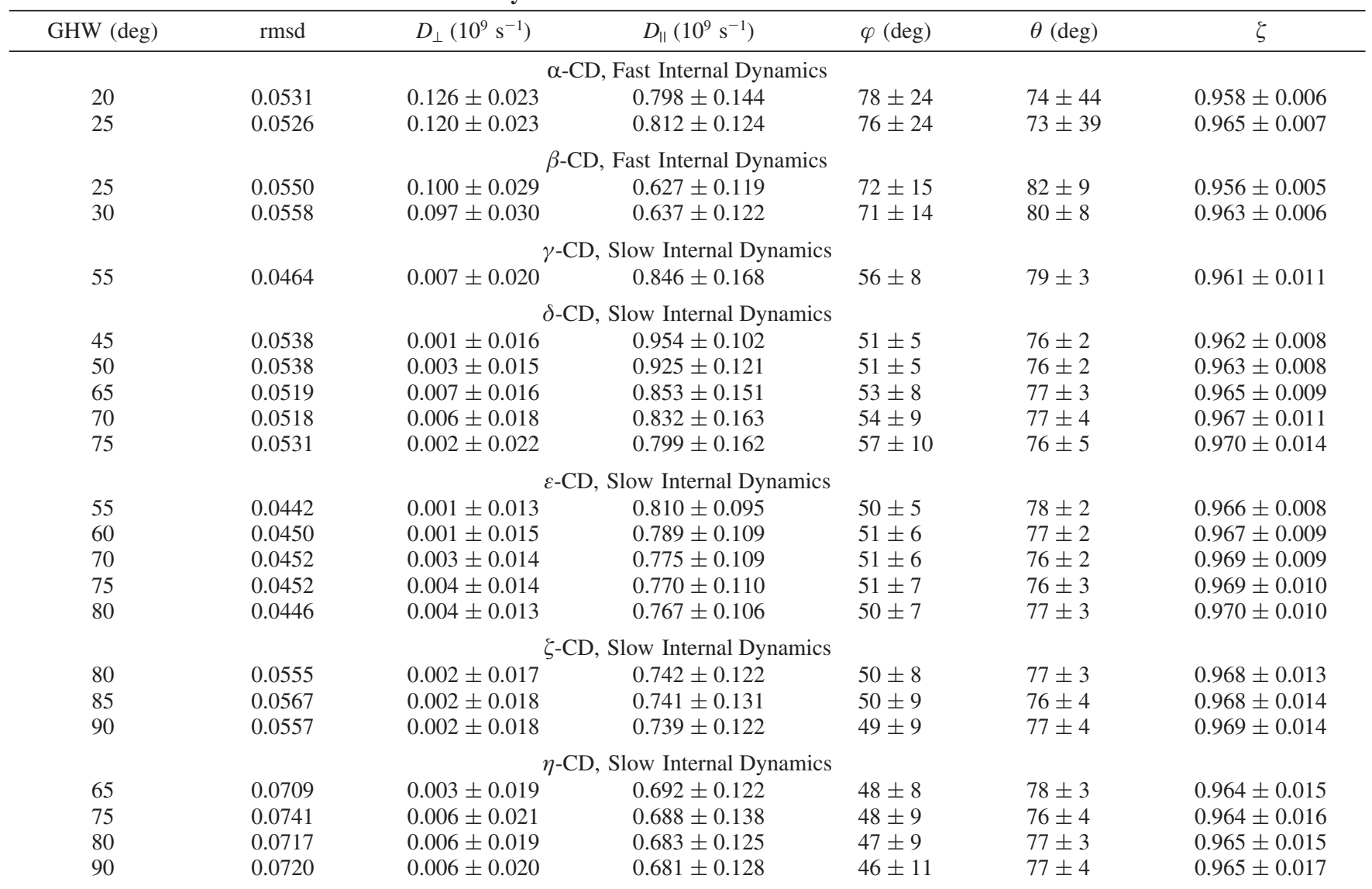

The iterative analyses were performed for each $\mathrm{CD}$, under assumptions of both slow and fast internal dynamics. The latter

is represented by the set of conformers, weighted by Gaussianshaped distributions, arising as a result of rotation of the glucopyranose unit about the axis connecting its terminal glycosidic oxygens. The widths at half-height of the Gaussian distributions, GHW, are varied from $5^{\circ}$ to $90^{\circ}$ with steps of $5^{\circ}$. This procedure yielded 36 preliminary results per CD.

Part of these results were rejected on the basis of the following criteria: (i) sets with negative RDT components, (ii) sets whose rmsd was higher by $5 \%$ or more in comparison to the best rmsd for a given $\mathrm{CD}$, (iii) sets with vibrational correction higher than 0.97, (iv) sets where the angle between the axis connecting glycosidic oxygens and the $z$ axis of the RDT, $\theta$, is smaller than $60^{\circ}$. The limit of 0.97 in point iii was chosen somewhat arbitrarily. The vibrational correction of the one-bond $\mathrm{CH}$ dipolar coupling constant should be between 0.83 and $0.95 .{ }^{42-46}$ We are aware of the fact, however, that the present analysis neglects relaxation arising from sources other than modulation of the intramolecular ${ }^{13} \mathrm{C}-{ }^{1} \mathrm{H}$ dipolar interactions. It is also not able to account for relaxation caused by the internal dynamics, if it happens that, contrary to the assumptions, the rates of the latter processes are comparable to the magnitudes of the RDT components. For these reasons vibrational corrections higher than those mentioned in refs $42-46$ might be expected. On the other hand, ref 42 quotes the value of 0.97 as a vibrational correction caused by stretching vibrations, and being independent of the temperature, environment, and molecular structure. Therefore, this value seems to be a reasonable choice for its upper limit. The value of $60^{\circ}$ in condition iv is also somewhat arbitrary. Mentioned angles lower than a certain value lead to close van der Waals contacts of atoms belonging to neighboring glucopyranose units, but due to the vague definition of the van der Waals radii there is no justification for any specific value of this angle.

Sets of the results of numerical analyses, which passed the described selection procedure, are listed in Table 1. The RDT components and angles $\theta$ and $\varphi$ are also visualized in Figures 2 and Figure 3, respectively.

The consecutive columns of Table 1 contain the widths at half-height of Gaussian distributions weighting individual conformers about the $\mathrm{O} 1-\mathrm{O} 4$ axis (GHW), the rmsd of each iterative analysis, the $D_{\perp}$ and $D_{\|}$components of the RDTs, the tilt of the glucopyranose plane (defined by carbon atoms 1,3 , and 5) with respect to the $x y$ plane of $\mathrm{CD}(\varphi)$, the angle between the $z$ axis of the cyclodextrin RDT and the $\mathrm{O} 1-\mathrm{O} 4$ axis of the glucopyranose unit $(\theta)$, and the vibrational correction $(\xi)$. The angles $\varphi$ and $\theta$ were calculated from positioning angles $\alpha$ and $\beta$. The latter pairs of angles were used in the course of numerical calculations, but they are not listed here. Their values are not important for the reader since they cannot be interpreted without the knowledge of the initial orientation of the rotated glucopyranose unit in the RDT coordinate frame.

First, let us notice that the analyzed relaxation data are reproduced well by the model of symmetric top. The highest departure of the best fit value from the experimental one of about $15 \%$ has been obtained for cross-relaxation at the highest field exploited, i.e., 11.7 T. This happens since at higher magnetic fields the cross-relaxation rates are smaller compared to those at lower fields, so that the relative error of the measurement is larger. In other cases the discrepancies are about $4-5 \%$. Inspection of Table 1 and Figure 2 reveals that the motional behavior of CDs does not change smoothly along with the number of glucopyranose units, $n$, in the CD cycle. $\mathbf{1}$ and $\mathbf{2}$ seem to depart significantly from the trend exhibited by the higher analogues. NMR data of the former molecules are better 


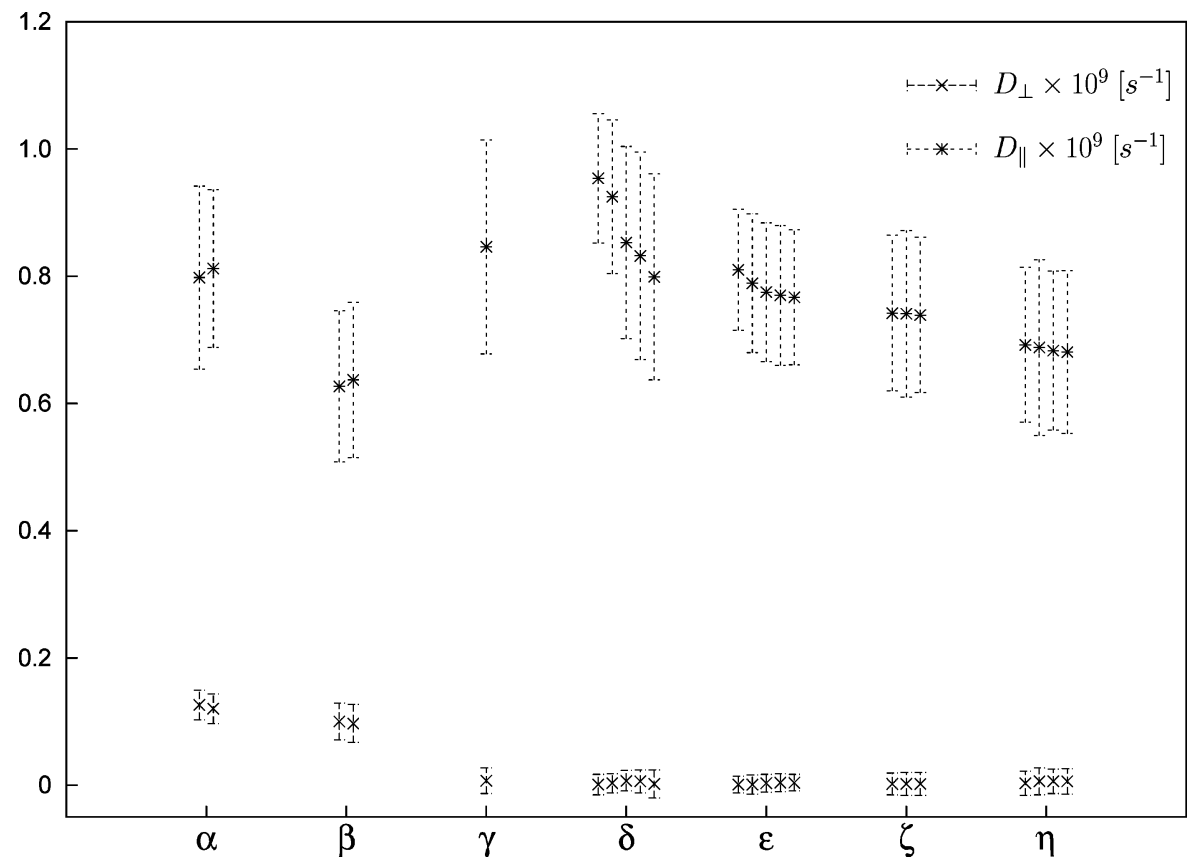

Figure 2. $D_{\perp}$ and $D_{\|}$components of RDTs for cyclodextrins 1-7. The data are taken from columns 3 and 4 of Table 1 . Error bars correspond to standard deviations.

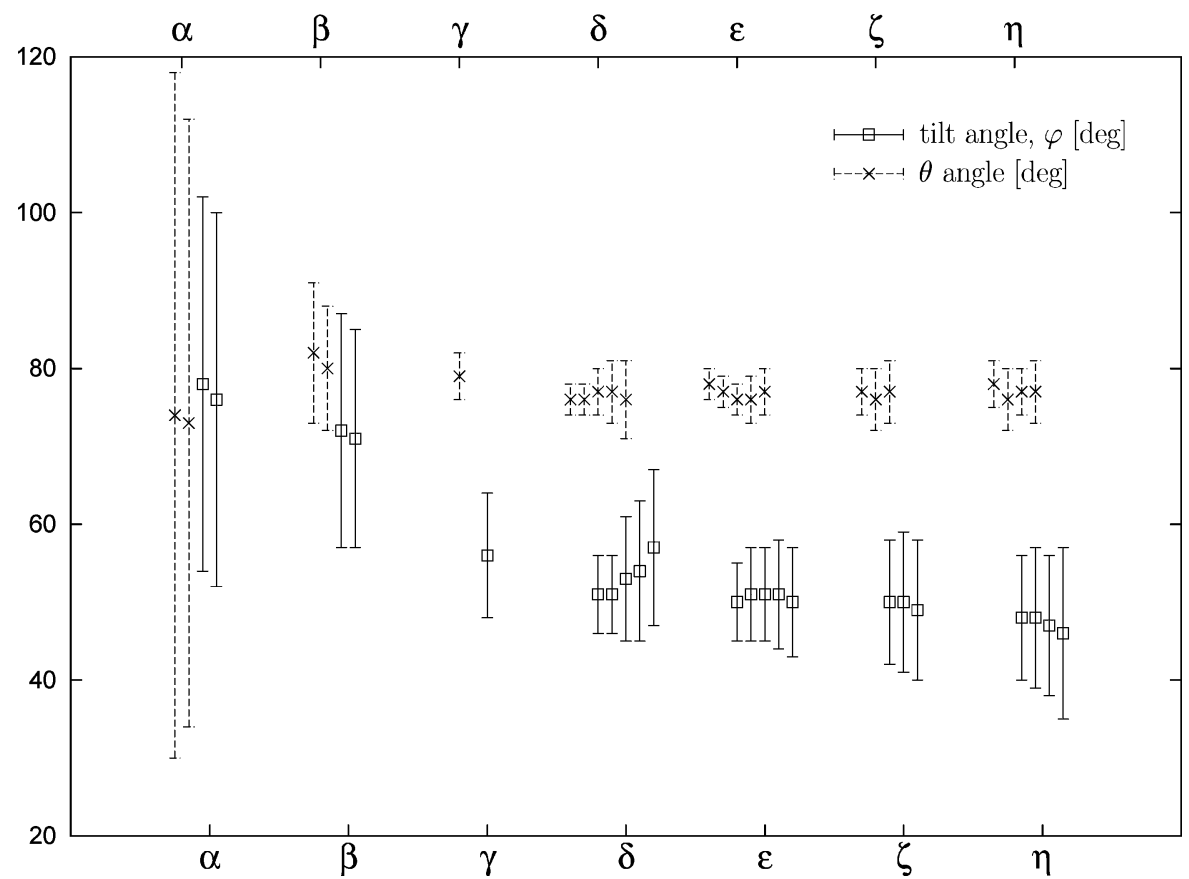

Figure 3. Angles $\theta$ and $\varphi$ for cyclodextrins 1-7. The data are taken from columns 5 and 6 of Table 1. Error bars correspond to standard deviations. For the sake of clarity the upper $x$ axis $(\theta$ angle) is slightly shifted with respect to the lower one ( $\varphi$ angle).

reproduced by the model of fast local dynamics, while for the latter ones the model of slow local motion works better. The GHW parameter is considerably lower for $\mathbf{1}$ and $\mathbf{2}$ than for the other CDs. This means that they tumble as rotors having a specific geometry while the geometries of larger CDs are "diffused" on the time scale of molecular tumbling. This is consistent with the intuitive belief that the ring-closing condition is much more restrictive for small CDs, so that their internal movement is more constrained than that of the larger ones. The magnitudes of the $\varphi$ angle are consistent with this picture too. The molecular geometry becomes more diffused, and the averaged tilt angle of the glucopyranose unit relative to the $x y$ plane of the respective RDT becomes smaller for larger CDs.
It is noteworthy that in all CDs the $\theta$ angle departs from $90^{\circ}$. Thus, in agreement with the CDs' nonrigidity discussed in the Introduction, the glycosidic oxygen atoms are on average not coplanar. This statement is somewhat weakened by significant standard errors for the $\theta$ angle in $\mathbf{1}$ and $\mathbf{2}$, but seems to be unquestionable for the remaining cycles. The mentioned large errors might stem from the possibility that the condition of fast local dynamics may not be strictly fulfilled for smaller CDs. Under such circumstances, the local dynamics would be a source of "extraneous" relaxation processes, which were not taken into account in the course of our analysis. For this reason we consider our results to be semiquantitative. This undescribed relaxation mechanism, together with neglected CSA relaxation, is a 
TABLE 2: Comparison of the Determined Tilt Angles, $\varphi$, and Amplitudes of Glucopyranose Wagging, GHW, with Literature Data

\begin{tabular}{lccl}
\hline & ref 48 & ref $49^{a}$ & this paper \\
\hline \multicolumn{4}{c}{ Tilt Angle $(\varphi)(\mathrm{deg})$} \\
$\alpha-\mathrm{CD}$ & $13 \pm 12$ & $13 \pm 10$ & $13 \pm 24$ \\
$\beta-\mathrm{CD}$ & $13 \pm 13$ & $14 \pm 10$ & $18.5 \pm 15$ \\
$\gamma-\mathrm{CD}$ & $9 \pm 17$ & $19 \pm 9$ & $34 \pm 8$ \\
\multicolumn{5}{c}{ Wagging Amplitude (GHW) (deg) } \\
$\alpha-\mathrm{CD}$ & $24^{b}$ & $20-25$ \\
$\beta-\mathrm{CD}$ & $38^{b}$ & $25-30$ \\
$\gamma-\mathrm{CD}$ & $50^{b}$ & 55
\end{tabular}

${ }^{a}$ Data obtained in the solid state by the X-ray diffraction method. ${ }^{b}$ Determined approximately from Figure $7 \mathrm{~b}$ of ref 48 .

possible reason for slightly higher vibrational correction than could be expected for $\mathrm{CH}$ bonds. Inspection of the last column of Table 1 reveals that $\xi$ never falls below 0.95 while the values of $0.91-0.93$ would be more plausible.

Finally, the determined parameters were used to reproduce the $R_{1 \rho \mathrm{C}}$ data. Experimental and calculated values can be found in the Supporting information. The agreement is very good for $\mathbf{1}$ and 2, while for the other CDs the applied model clearly fails. One reason for this failure has already been mentioned above; in CDs 3-7, where the local dynamic is slower than the overall molecular tumbling, the accurate calculations would require unknown ${ }^{13} \mathrm{C}$ chemical shifts in individual conformers. Inspection of eqs 1-3 reveals, however, another possible reason for the discrepancies. $R_{1 \rho \mathrm{C}}$ is the unique analyzed relaxation rate which depends on the spectral densities at the $\omega_{1}$ frequency, which is at least 5 orders of magnitude smaller than $\omega_{\mathrm{H}}$ and $\omega_{\mathrm{C}}$. In CDs $3-\mathbf{7}$ the $D_{\|}$is 2 orders of magnitudes larger than $D_{\perp}$. The latter is also much smaller than the Larmor frequencies involved. This renders $R_{1 \mathrm{C}}$ and $\rho_{\mathrm{C}}$ insensitive to the exact value of $D_{\perp}$, which is then diminished in the course of the iterative fitting process and delivered by the numerical routine with a large standard error. $D_{\perp}$ is, however, in the range where its magnitude has a significant impact on spectral densities taken at frequencies in the vicinity of $\omega_{1}$, i.e., on $R_{1 \rho \mathrm{C}}$. This seems to be the main reason for the substantial discrepancies in the reproduction of $R_{1 \rho \mathrm{C}}$ values for CDs 3-7.

Finally let us remark that the relaxation behavior of the nuclei within the methylene groups must be additionally influenced by rotation about the $\mathrm{C} 5-\mathrm{C} 6$ bond. The latter process does not appear to interfere much with the local dynamics considered above. Accordingly, the relaxation data for the nuclei in these side chains were not included in the analysis to avoid needless complications of the problem addressed presently.

\section{Conclusions}

The nuclear spin relaxation data for CDs built of 6-12 glucopyranose units were investigated. The numerical iterative analysis performed under the assumption of axially symmetric RDTs described reasonably the results obtained. The RDT components were determined along with the angles characterizing the spatial arrangement of glucopyranose units in the RDT coordinate frame. The components of RDTs do not change smoothly with the CD size. $D_{\perp}$ of $\mathbf{1}$ and $\mathbf{2}$ does not fit the trend formed by larger CDs. The same seems to be true for $D_{\|}$, but due to substantial errors which bear its values this statement is somewhat weakened in the case of $\mathbf{1}$. As expected, ${ }^{2,3}$ none of the CDs occur in liquids as a rigid truncated-cone structure. The internal motion in $\mathbf{1}$ and $\mathbf{2}$ seems to be faster than the overall molecular tumbling, while for larger CDs the opposite is true. The present observations seem to be consistent with the results of molecular dynamics (MD) calculations by Naidoo et al., 47,48 who concluded in ref 47 that $\mathbf{2}$ "undergoes small amplitude fast librations" and "has a tendency to highly increase the local water structure in the cavity and around the molecule" much more than $\mathbf{1}$ and $\mathbf{3}$. The values of the $\varphi$ angle (which is equal to $90^{\circ}$ $-\theta$ in Naidoo's notation) and GHW parameters extracted from Figure $7 \mathrm{~b}$ of ref 48 (see Table 2) also agree well with the present results.

Acknowledgment. Financial support from the scientific network "New applications of the nuclear magnetic resonance spectroscopy in chemistry, biology, pharmacy and medicine" is gratefully acknowledged.

Supporting Information Available: Experimental and calculated values of NMR relaxation parameters and the assignments of ${ }^{1} \mathrm{H}$ and ${ }^{13} \mathrm{C}$ NMR signals. This information is available free of charge via the Internet at http://pubs.acs.org.

\section{References and Notes}

(1) Dodziuk, H. In Cyclodextrins and Their Complexes. Chemistry, Analytical Methods, Applications; Dodziuk, H., Ed.; Wiley-VCH: Weinheim, Germany, 2006.

(2) Dodziuk, H. J. Mol. Struct. 2002, 614, 33.

(3) Dodziuk, H. In ref 1, Chapter 1.3.

(4) Harata, K. In ref 1, Chapter 7.2.

(5) Harata, K. In Comprehensive Supramolecular Chemistry; Szejtli, J., Ed.; Pergamon: Oxford, U.K., 1993; Vol. 3, Chapter 9.

(6) Eliel, E. L.; Allinger, N. L.; Angyal, S. J.; Morrison, G. A. Conformational Analysis; Interscience: New York, 1965.

(7) Dodziuk, H. In ref 1, Chapter 11.

(8) Ejchart, A.; Koümiński, W. In ref 1, Chapter 9.

(9) Steiner, T.; Moreira, A. M.; Texeira-Dais, J. J. C.; Müller, J.; Saenger, W. Angew. Chem., Int. Ed. 1995, 34, 1452.

(10) Jullien, J.; Cacenill, J.; Lacombe, L.; Lehn, J.-M. J. Chem. Soc., Perkin Trans. 2 1994, 989.

(11) Ellwood, P.; Spencer, C. M.; Spencer, N.; Stoddart, J. F.; Zarzycki, R. J. Inclusion Phenom. Mol. Recognit. Chem. 1992, 12, 121.

(12) Kowalewski, J.; Widmalm, G. J. Phys. Chem. 1994, 98, 28

(13) Ghalebani, L.; Kotsyubynskyy, D.; Kowalewski, J. J. Magn. Reson. 2008, 195,1 .

(14) (a) Lipari, G.; Szabo, A. J. Am. Chem. Soc. 1982, 104, 4546. (b) Lipari, G.; Szabo, A. J. Am. Chem. Soc. 1982, 104, 4559.

(15) Kowalewski, J.; Mäler, L. Nuclear Spin Relaxation in Liquids: Theory, Experiments, and Applications; Taylor and Francis: New York, 2006; Chapter 3.

(16) (a) Endo, T.; Ueda, H.; Kobayashi, S.; Nagai, T. Carbohydr. Res. 1995, 269, 369. (b) Endo, T.; Nagase, H.; Ueda, H.; Kobayashi, S.; Nagai, T. Chem. Pharm. Bull. 1997, 45, 532. (c) Miyazawa, I.; Ueda, H.; Nagase, H.; Endo, T.; Kobayashi, S.; Nagai, T. Eur. J. Pharm. Sci. 1995, 3, 153. (17) Wishart, D. S.; Bigam, C. G.; Yao, J.; Abildgaad, F.; Dyson, H. J.; Oldfield, E.; Markley, J. L.; Sykes, B. D. J. Biomol. NMR 1995, 6, 135.

(18) Farrow, N. E.; Muhandam, J. R.; Singer, A. U.; Pascal, S. M.; Kay, C. M.; Gish, G.; Shoelson, S. E.; Pawson, T.; Forman-Kay, J. D.; Kay, L. E. Biochemistry 1994, 33, 5984.

(19) Dayie, K. T.; Wagner, G. J. Magn. Reson., A 1994, 111, 121.

(20) Kay, L. E.; Nicholson, L. K.; Delaglio, F.; Bax, A.; Torchia, D. A. J. Magn. Reson. 1992, 97, 359.

(21) Canet, D. J. Magn. Reson. 1976, 23, 361.

(22) Delaglio, F.; Grzesiek, S.; Vuister, G. W.; Zhu, G.; Pfeifer, J.; Bax, A. J. Biomol. NMR 1995, 6, 277.

(23) Goddard, T. D.; Kneller, D. G. SPARKY 3; University of California: San Francisco.

(24) Fushman, D. In BioNMR in Drug Research; Zerbe, O., Ed.; WileyVCH: Weinheim, Germany, 2003; p 283.

(25) Van Geet, A. L. Anal. Chem. 1968, 40, 2227.

(26) Loening, N. M.; Keeler, J. J. Magn. Reson. 2002, 159, 55.

(27) Press, W. H.; Teukolsky, S. A.; Vetterling, W. T.; Flannery, B. P. Numerical Recipes: The Art of Scientific Computing; Cambridge University Press: New York, 2007; Chapter 9.

(28) Bernatowicz, P.; Kowalewski, J.; Szymański, S. J. Chem. Phys. 2006, 124, 024108 .

(29) Tropp, J. J. Chem. Phys. 1980, 72, 6035.

(30) Korzhnev, D. M.; Bileter, M.; Arsenie, A. S.; Orekhov, V. Y. Prog. NMR Spectrosc. 2001, 38, 197. 
Internal Dynamics in Cyclodextrins

(31) Luginbühl, P.; Wüthrich, K. Prog. NMR Spectrosc. 2002, 40, 199. (32) Palmer, A. G., III. Chem. Rev. 2004, 104, 3623.

(33) Kempf, J. G.; Loria, J. P. Cell Biochem. Biophys. 2003, 37, 187.

(34) Chen, Y. Y.; Luo, S. Y.; Hung, S. C.; Chan, S. I.; Tzou, D. L. M. Carbohydr. Res. 2005, 340, 723.

(35) Czugler, M.; Geiger, G.; Stezowski, J. J. Z. Kristallogr. 1983, 162, 54.

(36) Zabel, V.; Saenger, W.; Mason, S. A. J. Am. Chem. Soc. 1986, 108,3664 .

(37) Saenger, W. Nature (London) 1979, 279, 343.

(38) Imamura, K.; Nimz, O.; Jacob, J.; Myles, D.; Mason, S. A.; Kitamura, S.; Aree, T.; Saenger, W. Acta Crystallogr., B 2001, 57, 833

(39) Le Bas, G.; Mason, S. A. Acta Crystallogr., B 1994, 50, 717.

(40) Steiner, T.; Mason, S. A.; Saenger, W. J. Am. Chem. Soc. 1990, 112,6184 .
J. Phys. Chem. B, Vol. $x x x$, No. $x x, X X X X \quad$ G

(41) Steiner, T.; Mason, S. A.; Saenger, W. J. Am. Chem. Soc. 1991, 516 $113,5676$.

(42) Henry, E. R.; Szabo, A. J. Chem. Phys. 1985, 82, 4753.

(43) Case, D. A. J. Biomol. NMR 1999, 15, 95.

(44) Ottiger, M.; Bax, A. J. Am. Chem. Soc. 1998, 120, 12334. 157, 171.

(46) Bernatowicz, P.; Kowalewski, J.; Sandström, D. J. Phys. Chem. A 523 2005, 109, 57.

(47) Naidoo, K. J; Chen, J. Y · Jansson, J. L. M. Widmalm, G.; 525 Maliniak, A. J. Phys. Chem. B 2004, 108, 4236.

(48) Naidoo, K. J.; Gamieldien, M. R.; Chen, J. Y.; Widmalm, G.; 527 Maliniak, A. J. Phys. Chem. B 2008, 112, 15151. 528

(49) Harata, K. Chem. Rev. 1998, 98, 1803.

JP9084734 530 